\title{
In Vitro Fertilization Outcomes Following Assisted Hatching of Embryos with Thick Zona Pellucida-A Prospective Randomized Study
}

\author{
Mara Koifman'1, Shirly Lahav-Baratz', Libby Shopen², Blais Idit', David Ishai', \\ Zofnat Wiener-Megnazi', Ron Auslender ${ }^{1,2}$, Martha Dirnfeld ${ }^{1,2}$ \\ ${ }^{1}$ Division of Fertility-In Vitro Fertilization, Department of Obstetrics and Gynecology, Carmel Medical Center, \\ Haifa, Israel \\ ${ }^{2}$ Faculty of Medicine, Technion, Haifa, Israel \\ Email: Mara koifman@clalit.org.il
}

Received 22 July 2014; revised 24 August 2014; accepted 12 September 2014

Copyright (C) 2014 by authors and Scientific Research Publishing Inc.

This work is licensed under the Creative Commons Attribution International License (CC BY).

http://creativecommons.org/licenses/by/4.0/

(c) (i) Open Access

\begin{abstract}
Purpose: Impaired hatching is associated with implantation failure following in vitro fertilization (IVF). Thickening or hardening of the zona pellucida (ZP) has been proposed as a factor in this impairment. We examined whether selective assisted hatching (AH) is beneficial with embryos having a thick ZP. Methods: This prospective, randomized controlled study was performed in the IVF unit of an obstetrics and gynecology department in a university-affiliated hospital. Only patients undergoing IVF and having a ZP thickness of $\geq 17 \mu \mathrm{m}$ measured in all their embryos were included. In the intervention group, AH was applied to all embryos, before their transfer. In the control group, embryos were transferred without AH. Implantation, clinical pregnancy and live birth rates were the study endpoints. Results: Both study arms were comparable in most baseline parameters. The two groups did not differ in implantation rates $(14.1 \%$ control vs. $8.92 \%$ intervention, odds ratio $(\mathrm{OR})=0.5974,95 \%$ confidence interval $(\mathrm{CI}) 0.325-1.1)$, clinical pregnancy rates $(36.7 \%$ vs. $25.8 \%$, OR $=0.6025,95 \%$ CI $0.274-1.325)$, or live birth rates $(25 \%$ vs. $18.9 \%$, OR = $0.7021,95 \%$ CI 0.291 - 1.691). Conclusions: Selecting embryos for AH by their ZP thickness as a sole parameter was not found to be beneficial and to improve IVF outcome.
\end{abstract}

\section{Keywords}

In Vitro Fertilization, Assisted Hatching, Zona Pellucida Thickness, Partial Zona Dissection, Implantation Rate 


\section{Introduction}

A key event for successful embryo implantation is the expansion and thinning of the zona pellucida (ZP) and embryonic hatching [1]. One of the suggested explanations for impaired hatching, which may result in failure to implant and achieve pregnancy, is thickening or hardening of the ZP. Studies in women undergoing in vitro fertilization (IVF) have shown that maternal factors such as advanced age and active or passive smoking correlate with a thicker ZP [1] [2]. Hormonal factors including basal follicle stimulating hormone (FSH) and pre-ovulatory estrogen levels are associated with thick ZP [1] [3], and some studies have reported a direct relationship between the number of human menopausal gonadotropin (hMG) ampoules given for ovarian stimulation and ZP thickness [3]. In addition, laboratory conditions can induce hardening or thickening of the zona [4], and cryopreservation may also have a similar effect [4].

The assisted hatching (AH) procedure, first introduced in 1990 by Cohen et al. [5], targets improvement in IVF implantation rates by thinning, drilling, or completely removing the ZP before embryo transfer. Several techniques were developed for this purpose, including mechanical partial zona dissection (PZD) and use of proteolytic enzymes, Tyrode acid solution, laser energy [6], and Piezo technology [7]. Many studies have been performed to examine the benefit of $\mathrm{AH}$, but conclusions are inconsistent. According to the American Society for Reproductive Medicine committee opinion from 2008 [6], the available published data do not support routine or universal application of AH in IVF cycles, but AH may be clinically useful in patients with a poor prognosis, including those with $\geq 2$ failed IVF cycles, poor embryo quality, and advanced maternal age ( $\geq 38$ years of age).

The benefit of AH in patients with thickened ZP has not been established. In 1992, in a prospective, randomized controlled study, Cohen et al. [8] demonstrated a significant increase in implantation and clinical pregnancy rates with selective AH application, including in embryos with thickened ZP. Since then, several studies aimed to determine the effect of AH on embryos with thickened ZP but showed no significant improvement in IVF outcomes [9]-[11]. In a recent systematic review [12] including 28 studies and 5507 participants, AH was related to increased clinical pregnancy rates in women with repeated IVF failures or frozen-thawed embryos, but on the other hand, AH did not increase clinical pregnancy rates in non-poor prognosis patients or women with advanced age. In most of these studies, ZP thickness was the only one of several parameters analyzed for influence on the success of $\mathrm{AH}$, mainly the age of the women and repeated previous failures. However, no proper conclusions could be drawn regarding live birth.

In the present prospective randomized study, we included only patients whose embryos had a thickened ZP to determine whether selective $\mathrm{AH}$ is beneficial in this population. For this purpose, we compared implantation, pregnancy, and live birth rates between the intervention group whose embryos had undergone $\mathrm{AH}$ and a control group in which $\mathrm{AH}$ was not performed.

\section{Materials and Methods}

\subsection{Patient Recruitment}

The study was approved by the Committee for Research in Human Subjects of Carmel Medical Center. For study inclusion, the patients had to have all embryos with a ZP thickness of $17 \mu \mathrm{m}$ or more. Additional inclusion criteria were age 20 to 40 years and infertility due to male factors, mechanical factors, or both. The exclusion criterion was infertility due to any chronic illness, including endocrine disorders and endometriosis.

Patients were enrolled in the study during a 5-year period, between 2003 and 2008 and outcome follow up for frozen-thawed embryo transfer until 2012.

Each patient voluntarily signed an informed written consent before enrolling. Enrolled patients were randomly assigned either to the intervention group ( $\mathrm{AH}$ arm, 58 treatment cycles) or the control group (non-AH arm, 60 treatment cycles) using a standard random number table. Randomization was performed only after measurement of ZP thickness $>17$ um was found in all embryos.

The patients were not informed about the identity of their assigned group.

\subsection{Ovarian Stimulation and Ovulation Induction}

FSH levels on day 3 of the cycle were measured for each patient. Induction of ovulation was similar in all patients: down-regulation, using a long protocol, followed by ovarian stimulation as previously described by Dirnfeld et al. [13]. Briefly, gonadotropin releasing hormone analogue (Buserelin nasal spray, Suprefact, 1000 mg/day, Hoechst 
Pharmaceuticals, Israel) was administered in the midluteal phase and continued until human chorionic gonadotropin (hCG) administration (Ovitrelle $250 \mathrm{mcg}$, Merck Serono, Israel). Ovarian stimulation was performed using hMG (Menopur, Ferring, Israel, and/or rFSH (Puragon, MSD Israel or Gonal F, Merck Serono Israel)). Follicular growth monitoring was performed by transvaginal ultrasonography and measurement of serum levels of $17 \beta$-estradiol, luteinizing hormone, and progesterone.

After five or more follicles had reached a diameter of $17 \mathrm{~mm}$, patients received 250 mcg Ovitrelle, followed by ovum pick-up 35 - 37 hours later by transvaginal ultrasound-guided follicle aspiration. Luteal support was maintained by Vag. tablets Endometrin $100 \mathrm{mg}$ twice daily (Ferring, Israel).

\subsection{Fertilization}

The fertilization procedure, including AH, was done in the IVF center at Carmel Medical Center, Haifa. Fertilization techniques were performed using standard IVF or intracytoplasmic sperm injection (ICSI). Measurements of ZP thickness and AH procedures were carried out using a DiaPHOT 300 Nikon inverted microscope.

IVF was performed as previously described by Dirnfeld et al. [13] and ICSI as described by Van Steirteghem et al. [14] Fertilization was confirmed 16 to 20 hours after the procedure.

\subsection{ZP thickness Measurement}

ZP was prospectively measured by the same embryologist in all embryos on the day of embryo transfer by using an ocular micrometer scale. Two ZP measurements were performed by the same biologist for each of the embryos, and mean thickness was calculated.

If embryos were cryopreserved, ZP thickness was measured before cryopreservation. In all patients included in the present study, in both groups, all embryos had a ZP thickness $\geq 17 \mu \mathrm{m}$.

\subsection{Assisted Hatching}

AH was performed mechanically by partial zona dissection PZD just before embryo transfer to the uterine cavity. The cryopreserved embryos underwent the procedure after thawing and prior to the thawed embryo transfer.

AH was carried out on the Nikon microscope using Nomarski modulation optics with three-dimensional manipulators (Narishige, Tokyo, Japan). The procedure was performed as described by Feng et al. [15]. The embryo was placed in $5 \mu$ of cleavage medium supplemented with 10\% SSS under mineral oil (Sage, USA), held by a holding pipette. The PZD pipette (Cook, Australia) was advanced through the perivitelline space to impale the ZP. Then suction was discontinued from the holding pipette, and the microneedle impaling the ZP was brought below the holding pipette, which was rubbed over the impaled section of the ZP to create an incision.

\subsection{Embryo Transfer and Pregnancy Follow-Up}

Two embryos were selected for transfer into the uterine cavity and up to three embryos with repeated failures. The remaining embryos were cryopreserved. Cryopreservation and thawing were performed as previously described by Lahav-Baratz et al. [16]. Embryo transfer was performed on day 2 or 3 after ovum pickup, guided by transvaginal ultrasound. Embryo transfer was performed always on day 3 when egg collection was done on Thursdays. In other cases decision as to day 2 or day 3 transfer was taken at daily staff results meeting according to Clinician-Embryologist mutual convenience. The patients were not treated with corticosteroids or antibiotics.

Pregnancy was confirmed by blood $\beta$ HCG 12 days after embryo transfer and transvaginal ultrasound was performed at 4 - 6 weeks after embryo transfer. A clinical pregnancy was confirmed by observation of the gestational sac and positive fetal heart activity.

\subsection{Statistical Analysis}

Our primary outcome measure was clinical pregnancy rate, defined by the presence of a gestational sac with fetal heart activity by transvaginal ultrasound.

Other outcome measures were implantation rate (calculated as \# of gestational sacs visible on ultrasonography divided by total number of embryos transferred) and live birth rate.

Data analysis was conducted using SPSS 15.0. Quantitative data are presented as mean \pm standard deviation 
and categorical data as counts (percent). For evaluating the differences between groups, we used the chi-square test for categorical variables and t-test for continuous data. Multiple logistic regression analysis was used for multivariate analysis. A p $<0.05$ was considered statistically significant.

\section{Results}

A total of 118 IVF cycles of 109 infertile patients, enrolled from 2003 to 2008, were included in the study. The outcome follow-up for the frozen-thawed embryos continued until 2012.

Both of the study arms were comparable in terms of indications for IVF, number of previous treatment cycles, day 3 FSH levels, smoking habits, number of administered hMG ampoules, and mean ZP thickness (Table 1). The groups differed with respect to mean age (28.95 years, control group, vs. 30.81 years, AH group, $\mathrm{p}=0.045$ ). However, the study arms did not differ in other categorization of patients above or below 35 years old. Day 3 embryos were replaced in $46.7 \%$ of cycles in the control group, compared with $27.6 \%$ in the AH group (p $=0.032$ ). There was no difference in age or mean ZP thickness among patients treated with IVF compared to ICSI.

During the study period a total of 426 embryos were transferred (fresh + frozen). Clinical pregnancy was achieved in $35.1 \%$ of all treatment cycles and implantation rate was $11.5 \%$. A total of 26 pregnancies resulted in live birth (22\% per cycle), 22 of them singleton and 4 twins. Thirteen pregnancies ended in spontaneous pregnancy loss, and two resulted in preterm delivery at 20 and 25 weeks of gestation with no live birth.

Clinical pregnancy was the primary outcome (Table 2). A total of $36.7 \%$ of the patients in the control group, compared to $25.8 \%$ in the AH group, achieved a clinical pregnancy $(p=0.21)$. Implantation and live birth rates were higher in the control group but did not reach statistical difference (Table 2). With fresh embryo transfers, implantation rate was higher in the control group however, clinical pregnancy and live birth rates of did not differ between the study group and controls (Table 3). As for the frozen-thawed cycles, no significant difference was found in implantation, clinical pregnancy and live birth rates between the two study arms (Table 4).

With regard to $\mathrm{AH}$ within age groups, our findings revealed no significant difference in clinical pregnancy rates between the AH and control groups in patients $>35$ years old compared with the younger age group.

Analysis of factors other than AH that may independently affect pregnancy rate found that the timing of embryo replacement differed between the study and control group. A higher pregnancy rate was found when the embryos were transferred on day 3 compared with day 2 ( $43.2 \%$ vs. $24.3 \%$, respectively, $\mathrm{p}=0.033$ ).

Table 1. Comparison of clinical parameters of patients in the control group and A.H. group.

\begin{tabular}{|c|c|c|}
\hline Parameter & Group 1 (AH) & Group 2 (control) \\
\hline Number of cycles & 58 & 60 \\
\hline Age a & $30.81 \pm 4.94$ & $28.95 \pm 5.02$ \\
\hline$>35$ & $13(22.4 \%)$ & $7(11.6 \%)$ \\
\hline Previous failed IVF cycles & $2.43 \pm 2.94$ & $1.64 \pm 1.61$ \\
\hline Smokers & $10(17.2 \%)$ & $5(8.3 \%)$ \\
\hline \multicolumn{3}{|l|}{ IVF indications } \\
\hline Male & 47 (81.0\%) & $42(70 \%)$ \\
\hline Mechanical & $8(13.8 \%)$ & $13(21 \%)$ \\
\hline Combined & $3(5.2 \%)$ & $5(8.3 \%)$ \\
\hline Basal FSH level (IU/L) & $6.54 \pm 1.42$ & $6.43 \pm 1.84$ \\
\hline \multicolumn{3}{|l|}{ Embryo's age } \\
\hline 3 days $^{1}$ & $16(27.6 \%)$ & $28(46.7 \%)$ \\
\hline 2 days & $42(72.4 \%)$ & $32(53.3 \%)$ \\
\hline ZP thickness $(\mu \mathrm{m})$ & $22.1 \pm 2.16$ & $21.69 \pm 1.98$ \\
\hline No. of embryos replaced per transfer & $2.47 \pm 0.5$ & $2.53 \pm 0.5$ \\
\hline
\end{tabular}

Note: Mean values are given \pm SD. ${ }^{1} \mathrm{p}<0.05$. 
Table 2. Clinical outcome of all cycles, fresh and cryopreserved combined.

\begin{tabular}{cccc}
\hline Parameter & AH $(\mathrm{n}=58)$ & Control $(\mathrm{n}=60)$ & p value \\
\hline No. of embryos transferred & 213 & 213 & NS \\
Implantation $^{1}$ & $19 / 213(8.92 \%)$ & $30 / 213(14.1 \%)$ & NS \\
Positive $\beta$ hCG result $_{\text {Clinical pregnancies }}{ }^{2}$ & $25(43.1 \%)$ & $23(38.3 \%)$ & NS \\
Spontaneous abortions $_{\text {Live } \text { births }^{3} \text { (\% per cycle) }}^{15(25.8 \%)}$ & $23(38.3 \%)$ & $7(18.2 \%)$ & NS \\
\hline
\end{tabular}

Note: NS, not significant; ${ }^{1}$ The number of gestational sacs divided by total number of embryos transferred; ${ }^{2}$ Presence of gestational sac and fetal heart activity; ${ }^{3}$ Multiple birth is counted as one live birth.

Table 3. Clinical outcome of fresh embryo cycles.

\begin{tabular}{cccc}
\hline Parameter & $\mathrm{AH}(\mathrm{n}=58)$ & Control $(\mathrm{n}=60)$ & $\mathrm{p}$ value \\
\hline No. of embryos transferred & 143 & 152 & 0.048 \\
Implantation & $14 / 143(9.8 \%)$ & $27 / 152(17.8 \%)$ & NS \\
Clinical pregnancies & $11(18.9 \%)$ & $20(33.3 \%)$ & NS \\
Live births & $7(12.1 \%)$ & $13(21.6 \%)$ & \\
\hline
\end{tabular}

Note: NS, not significant.

Table 4. Clinical outcome of frozen embryo cycles.

\begin{tabular}{cccc}
\hline Parameter & AH $(\mathrm{n}=58)$ & Control $(\mathrm{n}=60)$ & $\mathrm{p}$ value \\
\hline No. of embryos transferred & 70 & 61 & $\mathrm{NS}$ \\
Implantation & $5 / 70(7.14 \%)$ & $3 / 61(4.91 \%)$ & NS \\
Clinical pregnancies & $4(18.2 \%)$ & $3(13.6 \%)$ & NS \\
Live births & $4(18.2 \%)$ & $2(9.1 \%)$ & \\
\hline
\end{tabular}

Note: NS, not significant.

\section{Discussion}

In the present prospective randomized study, we included only patients for whom all embryos had a ZP of $17 \mu \mathrm{m}$ and thicker. Our results have not shown any benefit, in terms of clinical pregnancy rates and live birth rates, to applying selective AH based on ZP thickness.

It took 5 years to find and randomize patients undergoing IVF with all their embryos with a ZP thickness of 17 $\mu \mathrm{m}$ or more and data for frozen-thawed cycles was available only by the end of 2012 .

This is the first study assessing the net effect of effect of AH when ZP thickness was the only parameter.

ZP thickness has been proposed as an important factor influencing implantation and pregnancy rates [1]. A thick ZP or impairment of its thinning as a result of in vitro culture conditions may inhibit embryo hatching and has been reported to jeopardize implantation and pregnancy achievement [1] [7]. This scenario has raised the question of whether AH could improve implantation and pregnancy rates among patients whose embryos have a thick ZP. If so, ZP thickness measurement could be routinely used as a more accurate tool for predicting AH benefit. Although patient's age in the study group was slightly higher than in the control group, we note that only ZP thickness and not age or previous repeated failures were used as criteria in selecting patients for $\mathrm{AH}$.

The results of fresh cycles showed a tendency, toward lower clinical pregnancy rates and lower implantation rate in the $\mathrm{AH}$ group however, this did not reach a statistical difference. Comparison of hatched and non-hatched frozen-thawed embryos did not show any benefit in performing AH based on ZP thickness. Although some previous studies showed benefit for applying AH to embryos of older patients, in the present study, we found no apparent advantage of $\mathrm{AH}$ in patients over age 35 years with a thick $\mathrm{ZP}$. We note that the later may have occurred due to the relatively small sample size of this age group. Interestingly, higher chemical pregnancy rate were observed in the AH group ( $p=0.038)$. Several studies have shown higher miscarriage rates following AH but had indistinct outcomes regarding the risk for miscarriage because of a lack of statistical power. Others reported a higher ectopic pregnancy rate associated with AH [17]. However, to our knowledge, no studies have found a correlation between $\mathrm{AH}$ and chemical pregnancies. Further investigation is needed to establish this association. 
Previous investigators have already reported that all methods currently available to create a gap in the ZP, including mechanical PZD, or Laser technology may have adverse effects on embryonic development [18]. In mice, zona drilling is associated with a high rate of cleavage arrest at the morula and blastocyst stages [11]. Further studies in humans also showed potential lethal damage to the embryo and to individual blastomeres, with reduction of embryo viability [4]-[7]. Another outcome might be loss of blastomeres or premature hatching during or after embryonic transfer as a result of contractions of the reproductive tract [18]-[20].

Moreover, blastomeres might become trapped in smaller holes during the hatching process and thus fail to complete hatching; alternatively, they may get lost through larger holes prior to the formation of tight junctions, possibly resulting in monozygotic twins and/or embryonic death [7]. Laser energy is now widely used because it appears to carry the lowest potential risk, and it is relatively simple to perform with consistency between operators [6]. Further studies using laser AH on embryos with thick ZP will provide additional information regarding possible benefit of this method in this embryo population.

We are aware of some limitations of this study. Despite randomization between AH and control groups, based on all embryos having ZP thickness of $\geq 17 \mu \mathrm{m}$, significantly more day 3 embryos were replaced in the controls as compared to the study group. We also found higher pregnancy rates when embryo transfer took place on day 3 compared to day 2, whether AH was performed or not. This may be one explanation to the higher pregnancy rates in the controls. This finding is consistent with the US Centers for Disease Control and Prevention data showing a slight increase in pregnancy rates with day 3 versus day 2 transfer, probably because the additional 24 hours in culture allows better embryo selection [21].

Another limitation is the small sample that has not allowed us to be more conclusive. However, finding patients with appropriate inclusion criteria with all embryos having a ZP of $\geq 17 \mu \mathrm{m}$ requested 5 years of patient recruitment.

\section{Conclusions}

In summary, the present study investigated the contribution of AH in a prospective randomized IVF/ICSI fresh and frozen-thawed cycles were all embryos that had a predefined thick ZP $(\geq 17 \mu \mathrm{m})$. Our findings showed that AH was not beneficial in embryos with a thick ZP.

Further studies with larger sample sizes using laser AH technology and single embryo transfer will contribute to a better understanding of the impact of AH on embryos with a thick ZP.

\section{Acknowledgements}

The authors thank Nili Stein for her contribution to the statistical analysis.

\section{References}

[1] Kilani, S.S., Cooke, S., Kan, A.K. and Chapman, M.G. (2006) Do Age and Extended Culture affect the Architecture of the Zona Pellucida of Human Oocytes and Embryos? Zygote, 14, 39-44. http://dx.doi.org/10.1017/S0967199406003625

[2] Shiloh, H., Lahav-Baratz, S., Koifman, M., Ishai, D., Bidder, D., Weiner-Meganzi, Z. and Dirnfeld, M. (2004) The Impact of Cigarette Smoking on Zona Pellucida Thickness of Oocytes and Embryos Prior to Transfer into the Uterine Cavity. Human Reproduction, 19, 157-159. http://dx.doi.org/10.1093/humrep/deh029

[3] Bertrand, E., Van Den Bergh, M. and Englart, Y. (1996) Clinical Parameters Influencing Human Zona Pellucida Thickness. Fertility and Sterility, 66, 408-411.

[4] Cohen, J. and Laufer, N. (1991) Assisted Hatching of Human Embryos. Journal of in Vitro Fertilization and Embryo Transfer, 8, 179-190. http://dx.doi.org/10.1007/BF01130802

[5] Cohen, J., Elsner, C., Kort, H., Malter, H., Massey, J. and Mayer, M.P. (1990) Impairment of the Hatching Process Following IVF in the Human and Improvement of Implantation by Assisting Hatching Using Micromanipulation. Human Reproduction, 5, 7-13.

[6] The Practice Committee of the Society for Assisted Reproductive Technology and the Practice Committee for Reproductive Medicine (2008) The Role of Assisted Hatching in in Vitro Fertilization: A Review of Literature. A Committee Opinion. Fertility and Sterility, 90, 196-198.

[7] Hammadeh, M.E., Fischer-Hammadeh, C. and Refaat Ali, K. (2011) Assisted Hatching in Assisted Reproduction: A State of the Art. Journal of Assisted Reproduction and Genetics, 28, 119-128. http://dx.doi.org/10.1007/s10815-010-9495-3 
[8] Cohen, J., Alikani, M., Trowbridge, J. and Rosenwaks, Z. (1992) Implantation Enhancement by Selective Assisted Hatching Using Zona Drilling of Human Embryos with Poor Prognosis. Human Reproduction, 7, 685-691.

[9] Balaban, B., Urman, B., Alatas, C., Mercan, R., Mumcu, A. and Isiklar, A. (2002) A Comparison of Four Different Techniques of Assisted Hatching. Human Reproduction, 17, 1239-1243. http://dx.doi.org/10.1093/humrep/17.5.1239

[10] Tucker, M.J., Luecke, N.M., Wiker, S.R. and Wright, G. (1993) Chemical Removal of the Outside of the Zona Pellucida of Day 3 Human Embryos Has No Impact on Implantation Rate. Journal of Assisted Reproduction and Genetics, 10, 187-191. http://dx.doi.org/10.1007/BF01239219

[11] Edirisinghe, W.R., Ahnonkitpanit, V., Promviengchai, S., Suwajanakorn, S., Pruksananonda, K. and Chinpilas, V. (1999) A Study Failing to Determine Significant Benefits from Assisted Hatching: Patients Selected for Advanced Age, Zonal Thickness of Embryos, and Previous Failed Attempts. Journal of Assisted Reproduction and Genetics, 16, 294301. http://dx.doi.org/10.1023/A:1020497714495

[12] Martins, W.P., Rocha, I.A., Ferriani, R.A. and Nastri, C.O. (2011) Assisted Hatching of Human Embryos: A Systematic Review and Meta-Analysis of Randomized Controlled Trials. Human Reproduction Update, 17, 438-453. http://dx.doi.org/10.1093/humupd/dmr012

[13] Dirnfeld, M., Goldman, S. and Gonen, Y. (1993) Functional Differentiation in Progesterone Secretion by Granulosa versus Cumulus Cells in the Human Pre-Ovulatory Follicle and the Effect of Different Induction of Ovulation Protocols. Fertility and Sterility, 60, 1025-1030.

[14] Van Steirteghem, A.C., Liu, J., Joris, H., Nagy, Z., Janssenswillen, C., Tournaye, H., et al. (1993) Higher Success Rate by Intracytoplasmic Sperm Injection than by Sub-Zonal Insemination. Report of a Second Series of 300 Consecutive Treatment Cycles. Human Reproduction, 8, 1055-1060.

[15] Feng, H.L., Hershlag, A., Scholl, G.M. and Cohen, M.A. (2009) A Retroprospective Study Comparing Three Different Assisted Hatching Techniques. Fertility and Sterility, 91, 1323-1325. http://dx.doi.org/10.1016/j.fertnstert.2008.02.133

[16] Lahav-Baratz, S., Koifman, M., Shiloh, H., Ishai, D., Wiener-Megnazi, Z. and Dirnfeld, M. (2003) Analyzing Factors Affecting the Success Rate of Frozen-Thawed Embryos. Journal of Assisted Reproduction and Genetics, 11, 444-448. http://dx.doi.org/10.1023/B:JARG.0000006705.46147.a2

[17] Chang, H.J. and Suh, C.S. (2010) Ectopic Pregnancy after Assisted Reproductive Technology: What Are the Risk Factors? Current Opinion in Obstetrics \& Gynecology, 22, 202-207. http://dx.doi.org/10.1097/GCO.0b013e32833848fd

[18] Stein, A., Rufas, O., Avrech, O., Pinkas, H., Ovadia, J. and Fisch, B. (1995) Assisted Hatching by Partial Zona Dissection of Human Preembryos in Patients with Recurrent Implantation Failure after in Vitro Fertilization. Fertility and Sterility, 63, 838-841.

[19] Frydman. N., Madoux, S., Hesters, L., Duvernoy, C., Feyereisen, E., Le Du, A., Tachdjian, G., Frydman, R. and Fanchin, R. (2006) A Randomized Double-Blind Controlled Study on the Efficacy of Laser Zona Pellucida Thinning on Live Birth Rates in Cases of Advanced Female Age. Human Reproduction, 21, 2131-2135. http://dx.doi.org/10.1093/humrep/del124

[20] Vajta, G., Holm, P., Greve, T. and Callesen, H. (1997) Survival and Development of Bovine Blastocysts Produced in Vitro after Assisted Hatching, Vitrification and In-Straw Direct Rehydration. Journal of Reproduction and Fertility, 111, 65-70. http://dx.doi.org/10.1530/jrf.0.1110065

[21] Paulson, R., Barbieri, R.L. and Barss, V.A. (2011) In Vitro Fertilization. http://uptodate.com/contents/in-vitro-fertilization 
Scientific Research Publishing (SCIRP) is one of the largest Open Access journal publishers. It is currently publishing more than 200 open access, online, peer-reviewed journals covering a wide range of academic disciplines. SCIRP serves the worldwide academic communities and contributes to the progress and application of science with its publication.

Other selected journals from SCIRP are listed as below. Submit your manuscript to us via either submit@scirp.org or Online Submission Portal.
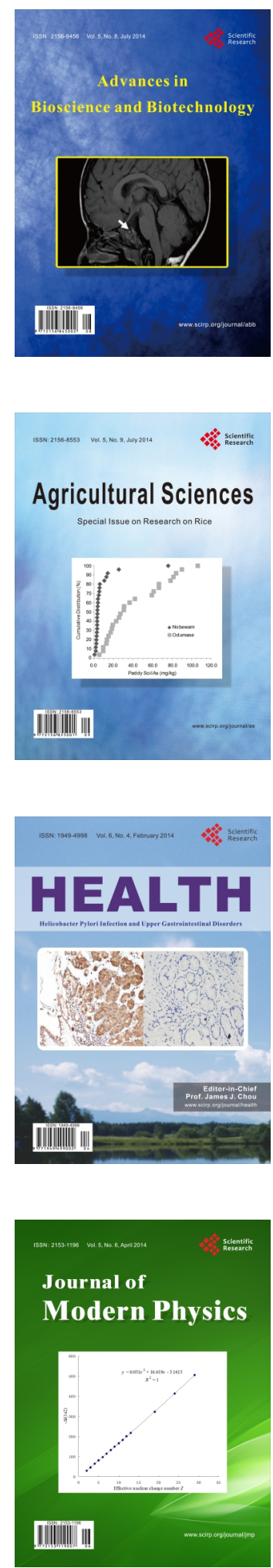
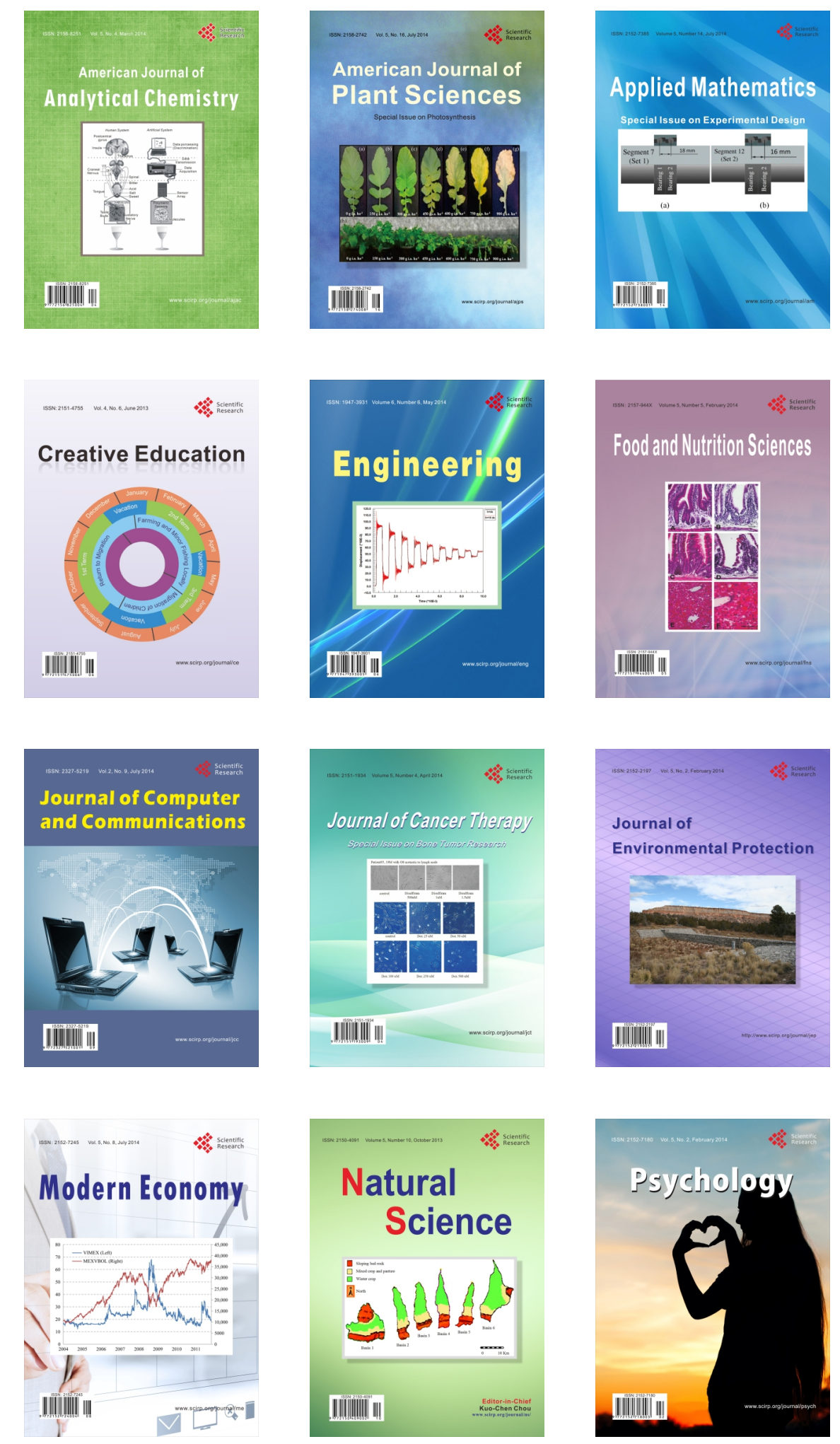